\title{
PICARD DIMENSIONS OF CLOSE TO ROTATIONALLY INVARIANT DENSITIES
}

\author{
TOSHIMASA TADA
}

\begin{abstract}
The purpose of this paper is to show that the Picard dimensions of a rotation-free density $P$ and a general density $Q$ on the punctured unit disk $0<|z|<1$ are equal to each other if $|P(z)-Q(z)|=O\left(|z|^{-2}\right)$ as $z \rightarrow 0$.
\end{abstract}

Before stating our result we fix terminologies. We denote by $\Omega$ the punctured unit disk $0<|z|<1$ which is viewed as an end of the punctured sphere $0<|z| \leq \infty$ so that the unit circle $|z|=1$ is the relative boundary $\partial \Omega$ of $\Omega$ and the origin $z=0$ is the ideal boundary $\delta \Omega$ of $\Omega$. By a density $P$ on $\Omega$ we mean a nonnegative locally Hölder continuous function $P$ on the closure $\bar{\Omega}=\Omega \cup \partial \Omega$ of $\Omega$ so that $P$ may or may not have a singularity at $z=0$. With a density $P$ on $\Omega$ we associate the class $P P(\Omega ; \partial \Omega)$ of nonnegative continuous functions $u$ on $\bar{\Omega}$ such that $u$ satisfies the elliptic equation

$$
\Delta u=P u, \quad \Delta=\partial^{2} / \partial x^{2}+\partial^{2} / \partial y^{2},
$$

on $\Omega$ and vanishes on $\partial \Omega$. We also denote by $P P_{1}(\Omega ; \partial \Omega)$ the subclass of $P P(\Omega ; \partial \Omega)$ consisting of functions $u$ with the normalization $u(1 / 2)=1$.

The Choquet theorem (cf., e.g., [7]) yields that there exists a bijective correspondence $u \leftrightarrow \mu$ between the convex set $P P_{1}(\Omega ; \partial \Omega)$ and the set of probability measures $\mu$ on the set ex. $P P_{1}(\Omega ; \partial \Omega)$ of extreme points of $P P_{1}(\Omega ; \partial \Omega)$ such that

$$
u=\int_{\mathrm{ex} . P P_{1}(\Omega ; \partial \Omega)} v d \mu(v)
$$

Thus the set ex. $P P_{1}(\Omega ; \partial \Omega)$ is essential for the class $P P_{1}(\Omega ; \partial \Omega)$, and the cardinal number \# (ex. $\left.P P_{1}(\Omega ; \partial \Omega)\right)$ of ex. $P P_{1}(\Omega ; \partial \Omega)$ is referred to as the Picard dimension of a density $P$ on $\Omega$ at the ideal boundary $\delta \Omega$ of $\Omega$, in $\operatorname{short} \operatorname{dim} P$, i.e.

$$
\operatorname{dim} P=\#\left(\operatorname{ex} . P P_{1}(\Omega ; \partial \Omega)\right) .
$$

If $\operatorname{dim} P=1$, then we say that the Picard principle is valid for $P$. In the study of the Picard principle the density $P_{0}(z)=|z|^{-2}$ plays an important role. For example the Picard principle for the density $P_{\lambda}(z)=|z|^{-2+\lambda}$ is valid if and only if $\lambda \in[0, \infty]$ $[3,4]$, the Picard principle for a density $P$ is valid if $P(z)=O\left(|z|^{-2}\right)$ as $z \rightarrow 0[2]$, and the Picard principle for a density $Q$ with $P(z) \leq Q(z) \leq P(z)+C|z|^{-2}(z \in \Omega)$ for a positive constant $C$ and a rotationally invariant density $P$, i.e. a density $P$ satisfying $P(z)=P(|z|)(z \in \Omega)$, is valid if the Picard principle for $P$ is valid [8]. The purpose of this paper is to show that the density $P_{0}(z)$ also plays an important

Received by the editors November 7, 1984 and, in revised form, May 14, 1986.

1980 Mathematics Subject Classification (1985 Revision). Primary 31A35; Secondary 30F25.

Key words and phrases. Picard dimension and principle, Martin boundary.

The author was supported in part by Grant-in-Aid for Scientific Research No. 59340007, Japanese Ministry of Education, Science, and Culture. 
role in the study of Picard dimensions by establishing the following theorem which is more general than the above results:

THEOREM 1. If $P$ is a rotationally invariant density on $\Omega$ and $Q$ is a general density of $\Omega$ with

$$
|P(z)-Q(z)|=O\left(|z|^{-2}\right) \quad \text { as } z \rightarrow 0,
$$

then we have $\operatorname{dim} P=\operatorname{dim} Q$.

We have seen that the range $\operatorname{dim} D$ of the mapping $P \mapsto \operatorname{dim} P$ from the set $D$ of densities on $\Omega$ into the set of cardinal numbers consists of all positive integers, the infinite countable cardinal number $\aleph_{0}$, and the cardinal number $\aleph$ of the continuum $[\mathbf{5}, \mathbf{6}]$ :

$$
\operatorname{dim} D=[1, \aleph]
$$

if the continuum hypothesis is postulated. In particular the range $\operatorname{dim} D_{r}$ of the set $D_{r}$ of rotationally invariant densities on $\Omega$ consists of 1 and $\aleph[3]$ :

$$
\operatorname{dim} D_{r}=\{1, \aleph\} .
$$

Then the above theorem shows that the range of the set of densities $Q$ on $\Omega$ with (1) for a rotationally invariant density $P$ on $\Omega$ also consists of 1 and $\aleph$.

We will recall in $\S 1$ the proof of (3) according to Nakai [3] and prove Theorem 1 in $\S 3$ by using the fundamental properties of "Martin kernels" for rotationally invariant densities $P$ on $\Omega$ with $\operatorname{dim} P=\aleph$ given in $\S \S 1$ and 2 .

The author is very grateful to Professor M. Nakai for his helpful suggestions.

1. Picard dimensions of rotationally invariant densities. 1. Consider a rotationally invariant density $P$ on $\Omega$. The unique bounded solution $e_{p}$ of $\Delta u=$ $P u$ on $\Omega$ with boundary values 1 on $\partial \Omega$ is referred to as the $P$-unit on $\Omega$. We also consider rotationally invariant densities $P_{n}(n=0,1, \ldots)$ on $\Omega$ defined by $P_{n}(z)=P(z)+n^{2}|z|^{-2}(z \in \bar{\Omega})$ and denote by $e_{n}$ the $P_{n}$-unit on $\Omega$, where we follow the convention $P_{0}=P$ and $e_{0}=e_{p}$. Then $e_{n}$ is positive and rotationally invariant on $\Omega$ and the function $e_{n}(r)$ of $r$ in $(0,1]$ is the unique bounded solution of

$$
l_{n} \phi(r) \equiv \frac{d^{2}}{d r^{2}} \phi(r)+\frac{1}{r} \frac{d}{d r} \phi(r)-P_{n}(r) \phi(r)=0 \quad(n=0,1, \ldots)
$$

on $(0,1)$ with boundary values 1 at $r=1$. Since $e_{n}(r) / e_{0}(r)$ is positive and increasing on $(0,1]$, there exists the limit

$$
\alpha_{n}(P)=\lim _{r \rightarrow 0} \frac{e_{n}(r)}{e_{0}(r)} \quad(n=1,2, \ldots)
$$

which is referred to as the $n$th singularity index of $P$ at $\delta \Omega$. In particular, we denote by $\alpha(P)$ the first singularity index $\alpha_{1}(P)$ and call it simply the singularity index of $P$ at $\delta \Omega$. Then we have the following fundamental inequality [3]:

$$
0 \leq \alpha(P)^{\left(3^{n}-1\right) / 2} \leq \alpha_{n}(P) \leq \alpha(P)^{n}<1 \quad(n=1,2, \ldots) .
$$

We also have the monotoneity of singularity indices [1]: for another rotationally invariant density $R$ on $\Omega$

$$
P \leq R \quad \text { implies } \quad \alpha(P) \leq \alpha(R) .
$$


2. We denote by $G_{P}(z, \varsigma)$ the $P$-Green's function on $\Omega$, i.e. the Green's function on $\Omega$ with respect to $\Delta u=P u$, and consider the function

$$
L_{P}(z, \varsigma)=\frac{G_{P}(z, \varsigma)}{e_{P}(\varsigma)} \quad(z, \varsigma \in \Omega) .
$$

In the case that $\alpha(P)=0$ the function $L_{P}(z, \varsigma)$ of $z$ converges to a minimal function $L_{P}(z)$ in $P P(\Omega ; \partial \Omega)-\{0\}$ as $\varsigma \rightarrow 0$ uniformly on every compact subset of $\Omega$, and hence we have

$$
\text { ex. } P P_{1}(\Omega ; \partial \Omega)=P P_{1}(\Omega ; \partial \Omega)=\left\{L_{P}(z) / L_{P}(1 / 2)\right\} \text {. }
$$

In the case that $\alpha(P)>0$ the function $L_{P}(z, \varsigma)$ of $z$ converges to a minimal function $L_{P}(z, \sigma)(\sigma \in[0,2 \pi))$ in $P P(\Omega ; \partial \Omega)-\{0\}$ as $|\varsigma| \rightarrow 0$ and $\arg \varsigma \rightarrow \sigma$ uniformly on every compact subset of $\Omega$. Furthermore, minimal functions $L_{P}(z, \sigma)$ are pairwise nonproportional, and

$$
\text { ex. } P P_{1}(\Omega ; \partial \Omega)=\left\{L_{P}(z, \sigma) / L_{P}(1 / 2, \sigma) ; \sigma \in[0,2 \pi)\right\}
$$

where we remark that

$$
\begin{gathered}
L_{P}(z, \sigma)=L_{P}\left(z e^{-i \sigma}, 0\right) \quad(z \in \Omega ; \sigma \in[0,2 \pi)), \\
L_{P}(z, 0)=L_{P}(\bar{z}, 0) \quad(z \in \Omega) .
\end{gathered}
$$

We then have the following characterization [3]:

$$
\operatorname{dim} P=1+\alpha(P) \cdot \aleph .
$$

2. Fundamental properties of $L_{P}(\cdot, 0)$. 1. In this section we consider a rotationally invariant density $P$ on $\Omega$ with $\alpha(P)>0$ and describe the properties of the function $L_{P}(\cdot, 0)$. We consider the $P$-Martin kernel $K_{P}(z, \zeta)=G_{P}(z, \zeta) / G_{P}(1 / 2, \zeta)$ $(z, \varsigma \in \Omega)$. As $|\zeta|, \arg \varsigma \rightarrow 0$ we obtain the $P$-Martin kernel $K_{P}\left(z, 0 e^{i 0}\right)$ with pole at $0 e^{i 0}$. The latter can be represented as $K_{P}\left(z, 0 e^{i 0}\right)=L_{P}(z, 0) / L_{P}(1 / 2,0)$.

Let $s$ be a positive number with $s<1$ and $\sigma, \tau$ be nonnegative numbers with $\sigma<\tau \leq \pi$. Then the function

$$
G_{P}(z, s)-G_{P}\left(z, s e^{i(\sigma+\tau)}\right)
$$

is the $P$-Green's function on

$$
\Omega_{\sigma, \tau}=\left\{|z|<1: \frac{\sigma+\tau}{2}-\pi<\arg z<\frac{\sigma+\tau}{2}\right\}
$$

with pole at $s$ so that we have

$$
G_{P}(z, s) \geq G_{P}\left(z, s e^{i(\sigma+\tau)}\right)
$$

and hence

$$
L_{P}(z, 0) \geq L_{P}(z, \sigma+\tau)
$$

for any $z$ in $\Omega_{\sigma, \tau}$. Since $\sigma<\tau$, the point $r e^{i \sigma}(r \in(0,1))$ is contained in $\Omega_{\sigma, \tau}$. Setting $z=r e^{i \sigma}$ in the above inequality, and using (6) yields $L_{P}\left(r e^{i \sigma}, 0\right) \geq L_{P}\left(r e^{-i \tau}, 0\right)$. Thus we obtain by (7)

$$
L_{P}\left(r e^{i \sigma}, 0\right) \geq L_{P}\left(r e^{i \tau}, 0\right) \quad(r \in(0,1], 0 \leq|\sigma| \leq|\tau| \leq \pi) .
$$


2. It is easy to see that the Fourier coefficient

$$
M_{P}(r)=\frac{1}{2 \pi} \int_{0}^{2 \pi} L_{P}\left(r e^{i \theta}, 0\right) d \theta
$$

of $L_{P}\left(r e^{i \theta}, 0\right)$ is a positive solution of $l_{0} \phi(r)=0$ on $(0,1)$ with vanishing boundary values at $r=1$. On the other hand the function

$$
E_{P}(r)=e_{P}(r) \int_{r}^{1} \frac{d s}{s e_{P}(s)^{2}}
$$

is also a positive solution of $l_{0} \phi(r)=0$ on $(0,1)$ with vanishing boundary values at $r=1$. Then there exists a positive constant' $\lambda_{P}$ such that $M_{P}(r)=\lambda_{P} E_{P}(r)$ and thus by (8)

$$
L_{P}(r, 0) \geq \lambda_{P} E_{P}(r) \quad(r \in(0,1])
$$

3. Proof of Theorem 1. 1. As a special case of condition (1) we assume in $\S \S 3.1-3.3$ that a rotationally invariant density $P$ on $\Omega$ and a general density $Q$ on $\Omega$ satisfy

$$
P(z) \leq Q(z) \leq P(z)+C|z|^{-2} \quad(z \in \Omega)
$$

for a positive constant $C$. When $\alpha(P)=0$ it was shown in [8] that $\operatorname{dim} P=$ $\operatorname{dim} Q=1$. We therefore also assume in $\S \S 3.1-3.3$ that $\alpha(P)>0$. Then we show in $\S 3.3$ that $\operatorname{dim} Q>\aleph_{0}$ by using auxiliary results in $\S \S 3.1-3.2$. The proof of Theorem 1 will then be completed in $\S 3.4$.

Take an integer $k$ with $k^{2}>C$ and consider the rotationally invariant density $R=P_{k}$ on $\Omega$, where as before

$$
P_{k}(z)=P(z)+k^{2}|z|^{-2} \quad(z \in \bar{\Omega}) .
$$

Then

$$
G_{P}(z, \zeta) \geq G_{Q}(z, \varsigma) \geq G_{R}(z, \varsigma), \quad e_{P}(\varsigma)=e_{0}(\varsigma) \geq e_{R}(\varsigma)=e_{k}(\varsigma)
$$

$(z, \varsigma \in \Omega)$, where $G_{Q}$ and $G_{R}$ are the $Q$-Green's and the $R$-Green's functions on $\Omega$, respectively. If we set

$$
m_{Q}=\min \left\{G_{Q}(1 / 2, \varsigma) ;|\varsigma|=1 / 4\right\}, \quad M_{Q}=\max \left\{G_{Q}(1 / 2, \varsigma) ;|\varsigma|=1 / 4\right\},
$$

then the maximum principle yields

$$
M_{Q} \frac{e_{P}(\varsigma)}{e_{P}(1 / 4)} \geq G_{Q}(1 / 2, \varsigma) \geq m_{Q} \frac{e_{R}(\varsigma)}{e_{R}(1 / 4)}
$$

for any $\varsigma$ with $0<|\varsigma|<1 / 4$. Therefore the $Q$-Martin kernel

satisfies

$$
K_{Q}(z, \varsigma)=\frac{G_{Q}(z, \varsigma)}{G_{Q}(1 / 2, \varsigma)}
$$

$$
\frac{e_{R}(1 / 4)}{m_{Q}} \frac{e_{P}(\zeta)}{e_{R}(\zeta)} L_{P}(z, \zeta) \geq K_{Q}(z, \zeta) \geq \frac{e_{P}(1 / 4)}{M_{Q}} \frac{e_{R}(\zeta)}{e_{P}(\zeta)} L_{R}(z, \zeta)
$$

$(z \in \Omega ; 0<|\zeta|<1 / 4)$.

2. Consider the Martin compactification $\Omega_{Q}^{*}$ of $\Omega$ with respect to the equation $\Delta u=Q u$ and the ideal boundary $\Gamma=\Gamma_{Q}=\Omega_{Q}^{*}-\Omega$. We denote by $\Gamma(\sigma)(\sigma \in$ $[0,2 \pi))$ the set of ideal boundary points $\varsigma^{*}$ such that there exists a sequence $\left\{\zeta_{n}\right\}_{1}^{\infty}$ 
in $\Omega$ with $\lim \left|\varsigma_{n}\right|=0, \lim \arg \varsigma_{n}=\sigma$, and $\lim \zeta_{n}=\varsigma^{*}$. Then $\Gamma$ is divided into the family $\{\Gamma(\sigma)\}: \Gamma(\sigma) \neq \varnothing, \Gamma=\bigcup\{\Gamma(\sigma) ; \sigma \in[0,2 \pi)\}$, with

$$
\Gamma(\sigma) \cap \Gamma(\tau)=\varnothing \quad(\sigma \neq \tau) .
$$

The above first and second properties are trivial and the last property (11) can be proved as follows:

Let $\zeta^{*}$ be any ideal boundary point in $\Gamma(\sigma)$ and let $\left\{\zeta_{n}\right\}_{1}^{\infty}$ be a corresponding sequence in $\Omega$ as above. Then $K_{Q}\left(z, \zeta_{n}\right)$ converges to the $Q$-Martin kernel $K_{Q}\left(z, \varsigma^{*}\right)$ with pole at $\varsigma^{*}$ as $n \rightarrow \infty$ uniformly on every compact subset of $\Omega$ and $e_{R}\left(\zeta_{n}\right) / e_{P}\left(\zeta_{n}\right)$ converges to $\alpha_{k}(P)$ as $n \rightarrow \infty$. Further $L_{R}\left(z, \zeta_{n}\right)$ converges to $L_{R}(z, \sigma)$ as $n \rightarrow \infty$ uniformly on every compact subset of $\Omega$ since $\alpha(R)>0$ by (5). Therefore by (10) $K\left(z, \varsigma^{*}\right)$ satisfies

$$
\frac{e_{R}(1 / 4)}{m_{Q}} \frac{1}{\alpha_{k}(P)} L_{P}(z, \sigma) \geq K_{Q}\left(z, \varsigma^{*}\right) \geq \frac{e_{P}(1 / 4)}{M_{Q}} \alpha_{k}(P) L_{R}(z, \sigma)
$$

$\left(z \in \Omega ; \varsigma^{*} \in \Gamma(\sigma) ; \sigma \in[0,2 \pi)\right)$, where by $(4) \alpha_{k}(P)>0$. Contrary to the assertion we suppose $\Gamma(\sigma) \cap \Gamma(\tau) \neq \varnothing$ for some $\sigma$ and $\tau$ with $\sigma \neq \tau$. Then the inequalities (12) for $\varsigma^{*}$ in $\Gamma(\sigma) \cap \Gamma(\tau)$ yield

$$
L_{P}(z, \sigma) \geq \beta L_{R}(z, \tau),
$$

where

$$
\beta=\beta(P, Q, k)=\frac{e_{P}(1 / 4)}{e_{R}(1 / 4)} \frac{m_{Q}}{M_{Q}} \alpha_{k}(P)^{2} .
$$

Therefore by (6) and (9) we have $L_{P}\left(r e^{i(\tau-\sigma)}, 0\right) \geq \beta \lambda_{R} E_{R}(r)$, and hence by (8)

$$
L_{P}\left(r e^{i \theta}, 0\right) \geq \beta \lambda_{R} E_{R}(r) \quad(r \in(0,1] ;|\theta| \leq d(\sigma, \tau)),
$$

where $d(\sigma, \tau)=\min (|\tau-\sigma|, 2 \pi-|\tau-\sigma|)$. Let $m=m(\sigma, \tau)$ be the minimum integer of the set of integers greater than $\pi / d(\sigma, \tau)$. Then by (6) and the inequalities

we have

$$
E_{R}(r) \geq \frac{e_{k}(r)}{e_{0}(r)} e_{0}(r) \int_{r}^{1} \frac{d s}{s e_{0}(s)^{2}} \geq \alpha_{k}(P) E_{P}(r)
$$

$$
\sum_{j=1}^{m} L_{P}\left(z, \frac{2(j-1)}{m} \pi\right) \geq \beta \lambda_{R} E_{R}(|z|) \geq \beta \lambda_{R} \alpha_{k}(P) E_{P}(|z|) \quad \text { for any } z \text { in } \Omega .
$$

Since $E_{P}(|z|)$ is a function in $P P(\Omega ; \partial \Omega)$ and every $L_{P}(\cdot, 2(j-1) \pi / m)(j=$ $1,2, \ldots, m)$ is a minimal function in $P P(\Omega ; \partial \Omega)$, there exist nonnegative constants $c_{1}, \ldots, c_{m}$ such that

$$
E_{P}(|z|)=\sum_{j=1}^{m} c_{j} L_{P}\left(z, \frac{2(j-1)}{m} \pi\right) .
$$

Thus we obtain by (6)

$$
\begin{aligned}
\sum_{j=1}^{m} c_{j} L_{P}\left(z, \frac{2 j-1}{m} \pi\right) & =\sum_{j=1}^{m} c_{j} L_{P}\left(z e^{-i \pi / m}, \frac{2(j-1)}{m} \pi\right) \\
& =E_{P}(|z|)=\sum_{j=1}^{m} c_{j} L_{P}\left(z \frac{2(j-1)}{m} \pi\right) .
\end{aligned}
$$


This contradicts the fact that every $L_{P}(z, j \pi / m)(j=0, \ldots, 2 m-1)$ is minimal.

3. We denote by $\Gamma_{0}$ the set of minimal points in $\Gamma=\Gamma_{Q}$. Then the Picard dimension of $Q$ is also given by the equality $\operatorname{dim} Q=\# \Gamma_{0}$ since

$$
\text { ex. } Q P_{1}(\Omega ; \partial \Omega)=\left\{K_{Q}\left(\cdot, \varsigma^{*}\right) ; \varsigma^{*} \in \Gamma_{0}\right\} \text {. }
$$

Let $\sigma\left(\varsigma^{*}\right)\left(\varsigma^{*} \in \Gamma_{0}\right)$ be the unique number in $[0,2 \pi)$ with $\varsigma^{*} \in \Gamma\left(\sigma\left(\varsigma^{*}\right)\right)$. If $\# \Gamma_{0} \leq$ $\aleph_{0}$, then there exist a number $\sigma_{1}$ in $[0,2 \pi)-\bigcup\left\{\sigma\left(\varsigma^{*}\right) ; \varsigma^{*} \in \Gamma_{0}\right\}$ and a point $\varsigma_{1}^{*}$ in $\Gamma\left(\sigma_{1}\right)$. The $Q$-Martin kernel $K_{Q}\left(\cdot, \zeta_{1}^{*}\right)$ with pole at $\zeta_{1}^{*}$ is represented in terms of $K_{Q}\left(\cdot, \varsigma^{*}\right)\left(\varsigma^{*} \in \Gamma_{0}\right)$ :

$$
K_{Q}\left(\cdot, \varsigma_{1}^{*}\right)=\sum_{\varsigma^{*} \in \Gamma_{0}} c\left(\varsigma^{*}\right) K_{Q}\left(\cdot, \varsigma^{*}\right)
$$

for nonnegative constants $c\left(\varsigma^{*}\right)$. Then the inequality

$$
K_{Q}\left(z, \varsigma_{1}^{*}\right) \geq c\left(\varsigma^{*}\right) K_{Q}\left(z, \varsigma^{*}\right) \quad(z \in \Omega)
$$

is valid for any $\varsigma^{*}$ in $\Gamma_{0}$ and thus by (12)

$$
L_{P}\left(z, \sigma_{1}\right) \geq \beta c\left(\varsigma^{*}\right) L_{R}\left(z, \sigma\left(\varsigma^{*}\right)\right) \quad\left(\varsigma^{*} \in \Gamma_{0}, z \in \Omega\right) .
$$

Now there exists a point $\varsigma_{2}^{*}$ in $\Gamma_{0}$ with $c\left(\varsigma_{2}^{*}\right)>0$ and so $\sigma_{1} \neq \sigma\left(\varsigma_{2}^{*}\right)$. This together with inequality (13) for $\varsigma^{*}=\varsigma_{2}^{*}$ yield a contradiction by the similar argument that led to the proof of (11). We therefore have proved the following result:

LEMMA 1. If $P$ is a rotationally invariant density on $\Omega$ and $Q$ is a general density on $\Omega$ with

$$
P(z) \leq Q(z) \leq P(z)+C|z|^{-2} \quad(z \in \Omega)
$$

for some positive constant $C$, then $\alpha(P)>0$ implies $\operatorname{dim} Q>\aleph_{0}$.

4. We now assume that the rotationally invariant density $P$ on $\Omega$ and the general density $Q$ on $\Omega$ satisfy (1). Then there exists a positive constant $C$ such that

$$
P(z)-C|z|^{-2} \leq Q(z) \leq P(z)+C|z|^{-2} \quad(z \in \Omega) .
$$

Consider the rotationally invariant density $R$ on $\Omega$ defined by

$$
R(z)=\max \left(P(z)-C|z|^{-2}, 0\right) \quad(z \in \bar{\Omega}) .
$$

Then $R$ satisfies

$$
\begin{aligned}
& R(z) \leq P(z) \leq R(z)+C|z|^{-2}, \\
& R(z) \leq Q(z) \leq R(z)+2 C|z|^{-2} \quad(z \in \bar{\Omega}) .
\end{aligned}
$$

Therefore, using Lemma $1, \alpha(R)=0$ implies $\operatorname{dim} P=\operatorname{dim} Q=1$ and $\alpha(R)>0$ implies $\operatorname{dim} P>\aleph_{0}, \operatorname{dim} Q>\aleph_{0}$. But by (3), $\operatorname{dim} P>\aleph_{0} i m p l i e s \operatorname{dim} P=\aleph$, and by $(2), \operatorname{dim} Q>\aleph_{0}$ implies $\operatorname{dim} Q=\aleph$ if the continuum hypothesis is postulated. Thus we conclude $\operatorname{dim} P=\operatorname{dim} Q$, and Theorem 1 is proved.

\section{REFERENCES}

1. H. Imai, On singular indices of rotation free densities, Pacific J. Math. 80 (1979), 179-190.

2. M. Kawamura, On a conjecture of Nakai on Picard principle, J. Math. Soc. Japan 31 (1979), 359-371. 
3. M. Nakai, Martin boundary over an isolated singularity of rotation free density, J. Math. Soc. Japan 26 (1974), 483-507.

4. - A test of Picard principle for rotation free densities, J. Math. Soc. Japan 27 (1975), 412-431.

5. __ The range of Picard dimensions, Proc. Japan Acad. 55 (1979), 379-383.

6. M. Nakai and T. Tada, The distribution of Picard dimensions, Kōdai Math. J. 7 (1984), 1-15.

7. R. Phelps, Lectures on Choquet's theorem, Math. Studies, No. 7, Van Nostrand, New York, 1965.

8. T. Tada, The role of boundary Harnack principle in the study of Picard principle, J. Math. Soc. Japan 34 (1982), 445-453.

Department of Mathematics, Daido institute of Technology, Daido, MiNAMI, NAGOYA 457 , JAPAN 